\title{
GEOMETRIC ACCURACY EVALUATION OF THE ZY-3 STEREO MAPPING SATELLITE FOR 8 YEARS
}

\author{
Liping Zhao* \\ Land Satellite Remote Sensing Application Center, Ministry of Nature Resource, Beijing, China - zhaolpwww@163.com
}

Commission I, WG I/10

KEY WORDS: ZY-3, Satellite, Stereo, Mapping, Accuracy

\begin{abstract}
:
The Ziyuan-3 (ZY-3) is the first civil high-resolution optical stereoscopic mapping satellite developed by China. It was launched on January 9, 2012, and as of January 9, 2020, it has been in a continuous operation for 8 years in orbit. The direct geolocation performances of ZY-3 based on the Rational Polynomial Coefficients (RPC) model are discussed, and the monoscopic and stereoscopic geometric accuracy of ZY-3 with ground control point (GCP) have also been studied using a bundle block adjustment with the affine correction in image space. Through the analysis of 47 sets of ZY-3 stereo data in Harbin, China, it can be found that from 2012 to 2019, the ZY-3 geolocation accuracy without GCP remains stable, with an average planimetric accuracy of approximately $16.2 \mathrm{~m}$, an average elevation accuracy of about $7.5 \mathrm{~m}$. The internal accuracy of the panchromatic triplet images is better than 1.0 pixel, the planimetric accuracy and elevation accuracy of stereo triplets and and stereo pairs are better than 2.0 meters with control points. In general, the results show that the ZY-3 satellite has been in good and stable condition during in-orbit operation in the past 8 years, and the various test indicators have been able to meet the design specifications.
\end{abstract}

\section{INTRODUCTION}

The Ziyuan-3 (ZY-3) is the first civil high-resolution optical stereoscopic mapping satellite independently developed by China. The mission is equipped with a set of panchromatic three-line array push-broom stereo cameras (TLC) and a $5.8 \mathrm{~m}$ spatial resolution multi-spectral camera (MSC) with a swath width of $51 \mathrm{~km}$. It is operated in a sun-synchronous and an altitude of $506 \mathrm{~km}$, with a 10:30 AM equator crossing time and an inclination of $97.421^{\circ}$. The two cameras with $3.5 \mathrm{~m}$ ground sample distance (GSD) mounted on the TLC point to the forward (FWD) and backward (BWD) at $22^{\circ}$ off-nadir angle, and the other camera with $2.1 \mathrm{~m}$ GSD points directly nadir (NAD). This solution has an excellent base-to-height ratio, which is beneficial to extract DEM, and can obtain thousands of kilometers of stereoscopic data by working continuously for a long time.

The ZY-3 project is mainly devoted to the operational application services of the stereo production of 1:50000 and larger scale maps, and provides data for natural resource investigation and monitoring, environmental surveying, emergency management, and national security needs.

It was launched on January 9, 2012, and as of January 9, 2020, it has been in a continuous operation for 8 years in orbit. The satellite has been in good condition and the data remains qualified.

In order to evaluate the geometric accuracy of the ZY-3 stereo mapping satellite, a total of 47 sets of ZY-3 stereo data are collected in the western region of Harbin, China from January 2012 to November 2019, and a set of high-precision reference data from ADS80 can be used for accuracy analysis.

Based on the Rational Polynomial Coefficients (RPC) model, the direct geolocation accuracy of ZY-3 is discussed, and using a bundle block adjustment with the affine correction in image space, the monoscopic and stereoscopic geometric accuracy of ZY-3 with GCP have also been studied.

\section{DATASETS}

In order to continuously evaluate and monitor the geometric performance of the ZY-3 stereo cameras, Harbin, Northeast China, with good weather conditions is selected as the experimental area.

A high-precision reference data set is available in the study area, and from January 2012 to November 2019, a total of 47 sets of ZY-3 data were collected. Based on the reference data, tiepoints are extracted using the phase correlation method, and they can be used as GCP or independent check points (ICP) for accuracy evaluation.

\subsection{Study area}

The study area is located in the northwest of the city of Harbin as shown in Figure 1. The main topographic features of the test area are flat, the terrain has very low undulations, the mean elevation is $160 \mathrm{~m}$, and the height difference is less than $70 \mathrm{~m}$ ranging from $140 \mathrm{~m}$ to $210 \mathrm{~m}$ above the WGS84 ellipsoid. Flat terrain facilitates the analysis of the geometric properties of the image itself, eliminating terrain effects as much as possible. In addition, the flat area can not only guarantee the accuracy of the reference data, but also ensure the accuracy of GCP / ICP automatic extraction and reliability.

The land coverage of the study area is simple, mainly with farmland and grassland, and there are some villages dotted between them, there are a few built-up areas, and lofty trees and buildings are scarce. The problem in this area is that the ground cover will change with the seasons, which will cause some difficulties in accurately extracting GCP / ICP.

\footnotetext{
* Corresponding author
} 
In general, due to good weather conditions, flat terrain, simple surface coverage, and small changes in the test area, choosing this area as the research area is conducive to long-term continuous monitoring of the geometric accuracy of the ZY-3 mapping satellite.

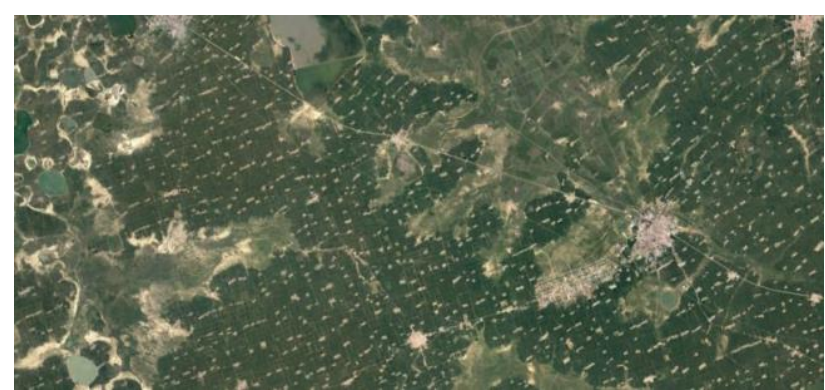

Figure 1. The schematic diagram of the study area

\subsection{Reference data}

In the study area, a high-precision reference data set is available. The data is rectangular shape, with an area of approximately $3600 \mathrm{~km}^{2}$, an east-west width of about $120 \mathrm{~km}$, and a northsouth length of $30 \mathrm{~km}$. The dataset is comprised of panchromatic ortho-image with $0.5 \mathrm{~m}$ ground sample distance (GSD) and a planimetric accuracy of $0.5 \mathrm{~m}$ RMSE, and digital surface model (DSM) with $2.0 \mathrm{~m}$ grid spacing and an accuracy better than $1.0 \mathrm{~m}$ RMSE. The panchromatic ortho-image and DSM are produced from ADS80 aerial imageries acquired in October 2014 using software such as X-Pro and INPHO.

\subsection{ZY-3 data}

In the region, from January 2012 to November 2019, a total of 47 sets of available ZY-3 stereo triplets with good quality were preferred for accuracy analysis. The data spans up to 8 years, covering different seasons and each month, with an average of about 6 sets of stereo data per year, with a minimum of 3 sets in

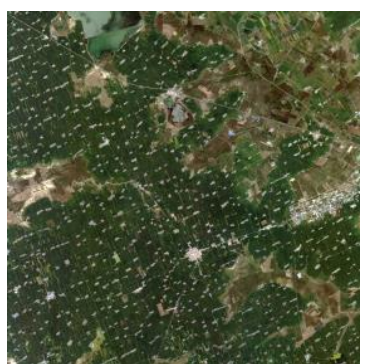

(a) ZY3_MS_20190914

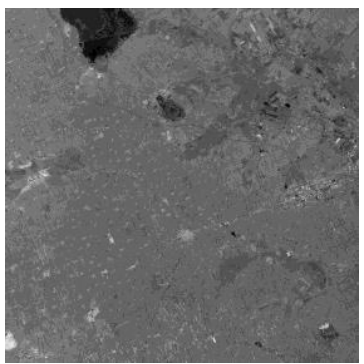

(c) ZY3_BWD_20190914

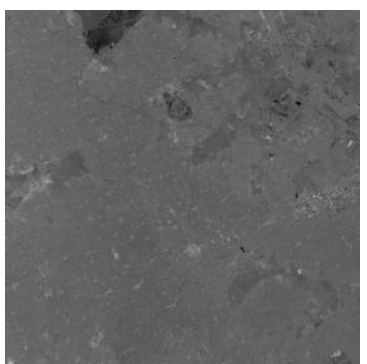

(b) ZY3_NAD_20190914

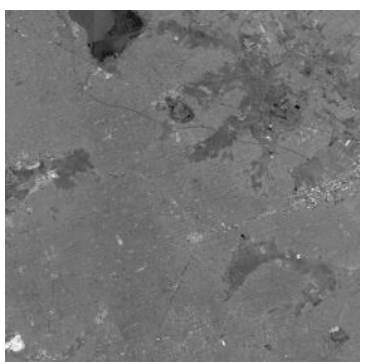

(d) ZY3_FWD_20190914
Figure 2. ZY-3 thumbnail images (20190914). (a) multi-spectral image; (b) panchromatic NAD image; (c) panchromatic BWD image; (d) panchromatic FWD image.
2014 and a maximum of 10 sets in 2016. Figure 2 shows the thumbnail of images data obtained on September 14, 2019. The off nadir look angle of these data in across track direction is less than $5^{\circ}$, which does not affect the accuracy evaluation.

\section{$2.4 \quad Z Y-3$ product}

A standard set of $\mathrm{ZY}-3$ products includes 4 scenes of image data, namely panchromatic nadir (NAD), backward (BWD), forward (FWD) images and multi-spectral image. Each image data is accompanied by a set of Rational Polynomial Coefficients as a geometric model. Table 1 and Table 2 summarize the main characteristics of the ZY-3 satellite and the panchromatic standard products, respectively.

\begin{tabular}{|c|c|}
\hline Launch date & January 9,2012 \\
\hline Design life & 5 years \\
\hline Pointing capability in cross-track & $\pm 32^{\circ}$ \\
\hline Altitude & $505 \mathrm{~km}$ \\
\hline Cycle & 59 days \\
\hline
\end{tabular}

Table 1. Main characteristics of the ZY-3

\begin{tabular}{|c|c|}
\hline Spectral range & $500-800 \mathrm{~nm}$ \\
\hline $\begin{array}{c}\text { FOV (Filed of } \\
\text { View) }\end{array}$ & $\geq 6^{\circ}$ \\
\hline Swath width & $\begin{array}{c}\text { Nadir: }>51 \mathrm{~km} \\
\text { Backward/Forward: }>52 \mathrm{~km}\end{array}$ \\
\hline Geolocation & $100 \mathrm{~m}$ without GCPs \\
25 m with GCPs
\end{tabular}

Table 2. Main characteristics of the panchromatic production

\subsection{GCP and ICP}

Fast Fourier Transform Phase Matching (FFTPM) (Kuglin and Hines, 1975; Foroosh et al., 2002) is used to extract tie points (TPs) between the stereo image data and reference data. FFTPM works in the frequency domain, it pays more attention to the texture information of the image and is a more robust image matching algorithm, which can not only overcome the difficulty of matching caused by the change of ground coverage of multitemporal images, but also eliminate the adverse effects brought by different image resolutions.

The difference in ground coverage and the size of the overlap range between the ZY-3 stereo data and the reference data will affect the number of TPs, resulting in different amounts of TPs that can be extracted from each set of stereo data. For all 47 sets of test data, after removing the gross errors, the number of TPs retained ranges from 3500 to 16,000 . Moreover, the points automatically extracted using the FFTPM algorithm is not only uniformly distributed (as shown in Figure 3 and Figure 4), but also has much higher accuracy than the points measured manually, so it can more objectively reflect the accuracy of ZY3 image data.

The affine correction in image space based on the rational polynomial coefficients (RPC) is chosen as an adjustment model to analyze the planimetric and altimetric accuracy of ZY3 stereo image data. In addition, since a large amount of TPs can be extracted for each set of test data, in order to avoid the adverse impact of the distinction between control points and check points on the accuracy evaluation, all the TPs are used as 
ground control points or independent checkpoints for accuracy evaluation.

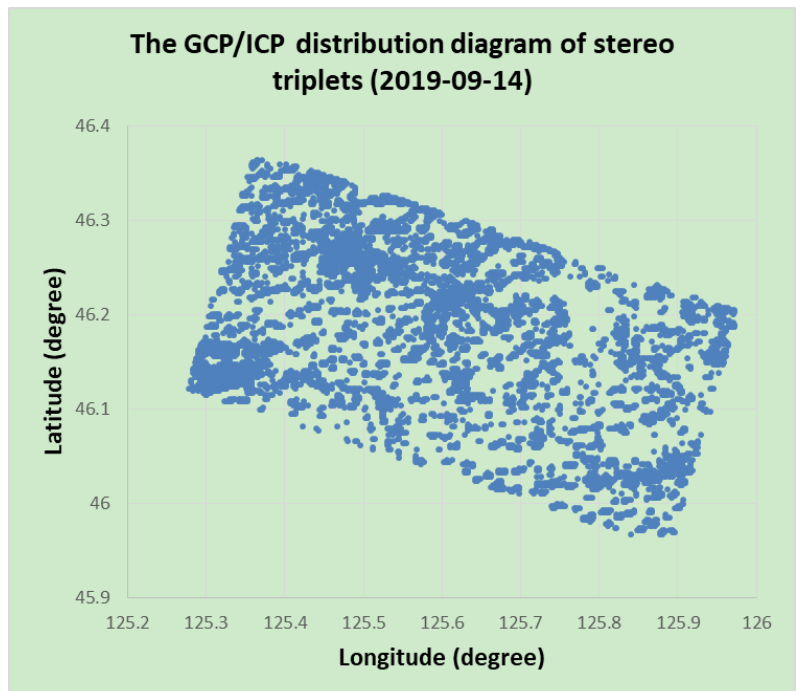

Figure 3. The GCP/ICP distribution diagram of stereo triplets (2019-09-14)

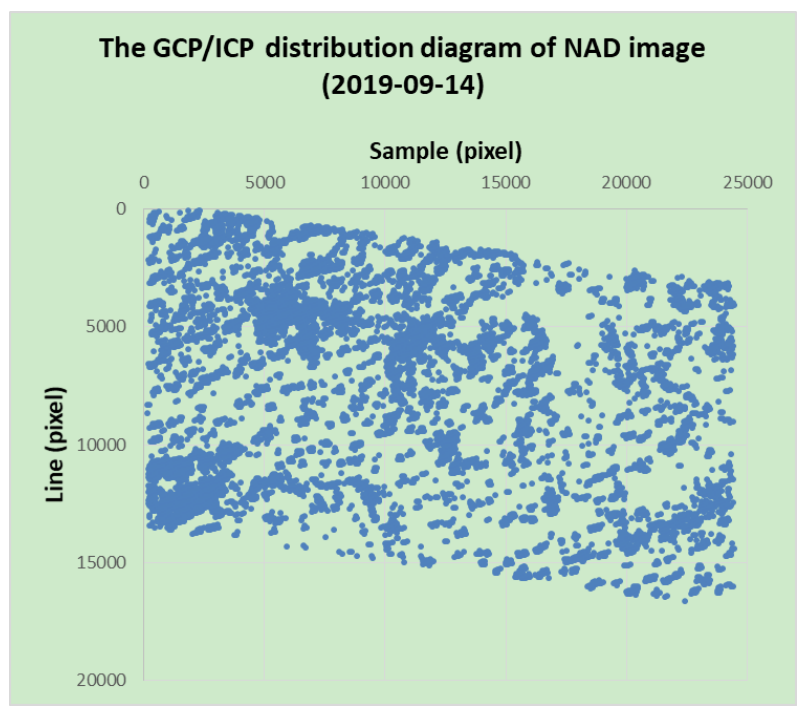

Figure 4. The GCP/ICP distribution diagram of NAD image (2019-09-14)

\section{GEOMETRY MODEL}

\subsection{Geometrical characteristics}

As a stereo mapping satellite, in order to meet the strict accuracy requirements, ZY-3 adopts metric design and precise internal geometric calibration (Cao et al., 2012). It is also required to equip the cameras with extremely high orbit accuracy, attitude accuracy, timing accuracy and stability. The cameras use a refractive quasi telecentric optical system in image space, which has a better thermal stability than other systems. The optomechanical structure of the titanium alloy is selected, and the high-precision thermal control technology is adopted to ensure the stable interior orientation elements and the minimal distortion (ZY-3A, 2020). The ZY-3 satellite calculates the precise orbit position through a dual-frequency GPS receiver on-board and a satellite laser reflector (SLR), and relies on the combination of multiple high-precision gyroscopes and star sensors to solve the precise attitude.

\subsection{Physical sensor model}

After completing the internal and external geometric calibration, using the orbital position determined by GPS, the attitude data obtained by the star trackers, and precise timing system, the physical sensor model of the cameras can be established to relate ground positions to image pixels.

\subsection{RPC model}

The physical sensor model is more complex and lacks supporting software, which brings many inconveniences to a wide range of applications. Usually, the RPC model is used to replace the strict physical model to establish the mapping relationship between ground objects and image pixels. The ratio of third-order polynomial equations is applied to fit the physical model by satellite data providers, and coefficients are given through the metadata file attached to the image. In this paper, the RPC model provided by the supplier is used to study the direct geolocation accuracy, and it is also used for the bundle adjustment with GCP to optimize geometry performance.

\section{DIRECT GEOLOCATION}

\subsection{Methodology}

The direct geolocation accuracy of mapping satellites, also known as absolute geolocation accuracy, is one of the main performance indicators of satellite missions. It is mainly affected by various factors such as camera, ephemeris, attitude, etc., as it will determine the number of ground control points needed to process satellite images and has a great impact on many applications.

For the ZY-3 standard stereo triplet products, the direct geolocation accuracy in the object space is evaluated using the RPC model without any adjustment, all automatically extracted tiepoints are all used as ICP. Among them, there are the following issues that needs to be explained:

1) The RPC model has imbedded information such as camera, ephemeris, and attitude, but there is no atmospheric refraction correction;

2) The ground reference coordinates come from the highprecision ortho image and DSM, so a large number of check points can be obtained by automatic matching, but gross errors need to be eliminated.

To estimate the absolute horizontal and vertical geolocation accuracy of the panchromatic ZY-3 stereo triplets, the classic following steps are performed:

1) Extract the ICP through the phase correlation method and remove gross errors, results in a list of the ground coordinates and image coordinates of the checkpoint;

2) Calculate the ground coordinates of the checkpoint based on the pixel position (line, sample) of the stereo intersection point of the NAD, BWD and FWD triple images;

3) For each checkpoint, subtract ground reference coordinates from triplet-imagery-derived ground coordinates, results in a list of " $\triangle$ Easting", " $\triangle$ Northing" and " $\triangle$ Height" values;

4) For each stereo triplet, compute the mean, standard deviations and RMS error of " $\triangle$ Easting", " $\triangle$ Northing" and " $\triangle$ Height" values;

5) Estimate the RMS error statistics for populations of stereo triplets, giving the time series trend of the direct geolocation of ZY-3 for 8 years. 


\subsection{Results and analysis}

Regarding the evaluation of the direct geolocation accuracy of ZY-3, this article only considers the planimetric accuracy and elevation accuracy of the stereoscopic triplets, and does not discuss the accuracy of monoscopic image.

Figure 5 depicts the direct geolocation accuracy in the three directions of north, east, and height in accordance with the time series. Figures 6 and 7 have statistics on horizontal accuracy and vertical accuracy, respectively.

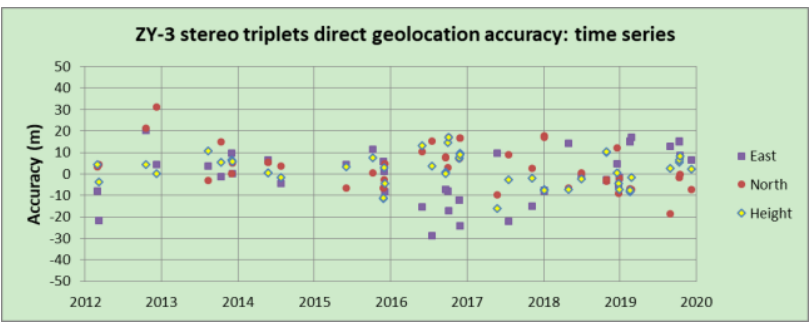

Figure 5. ZY-3 stereo triplets direct geolocation accuracy (time series).

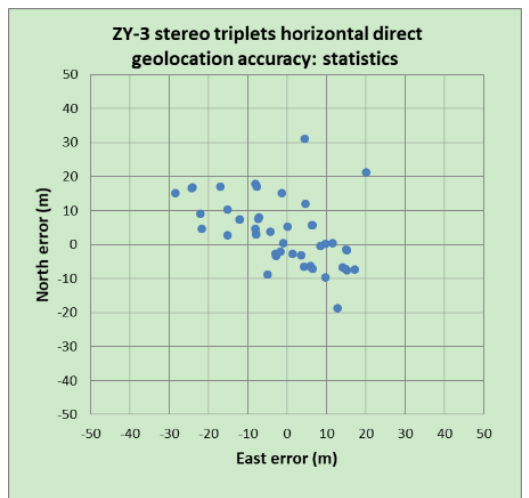

Figure 6. ZY-3 stereo triplets horizontal direct geolocation accuracy (statistics). Num=47 stereo triplets; Average $($ East $)=-$ $1.11 \mathrm{~m} ; \quad$ Average $($ North $)=3.400 \mathrm{~m} ; \quad \operatorname{RMS}($ East $)=12.431 \mathrm{~m}$; RMS $($ North $)=10.438 \mathrm{~m} ;$ RMS $($ Horizontal $)=16.232 \mathrm{~m}$.

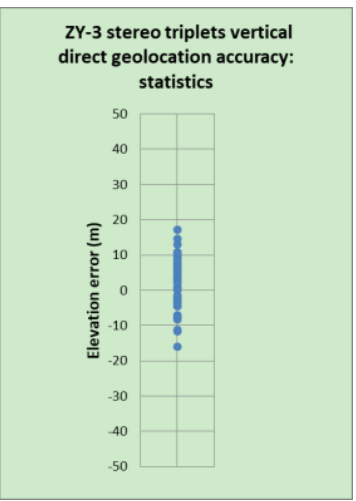

Figure 7. ZY-3 stereo triplets vertical direct geolocation accuracy (statistics). Num $=47$ stereo triplets; Average $=1.572 \mathrm{~m}$; $\mathrm{RMS}=7.518 \mathrm{~m}$.

Analyzing Figure 5, Figure 6 and Figure 7, it can be found that from 2012 to 2019, the ZY-3 geolocation accuracy without GCP remains stable, with an average planimetric accuracy of approximately $16.2 \mathrm{~m}$, an average elevation accuracy of about $7.5 \mathrm{~m}$, a maximum planimetric error of $32.4 \mathrm{~m}$ and a maximum elevation error of $17.2 \mathrm{~m}$, which satisfies the satellite design specifications.

\section{ADJUSTMENT WITH GCP}

\subsection{Methodology}

The internal geometric accuracy, that is the adjustment accuracy with GCP, is a very important basic performance of a mapping satellite, and it is also one of the key criterias for the operating status of satellites, mainly reflecting the internal geometric calibration accuracy of camera. It has important influence on many applications such as resource investigation and monitoring, disaster warning and reduction, water conservation, and ecological environment.

In order to evaluate the internal geometric accuracy of the ZY-3 stereo triplets and stereo pairs, a bundle block adjustment with GCP is performed, and the affine correction in image space is estimated according to the previous experience (Grodecki, 2001; Jacobsen, 2007; Zhao et al., 2018).

Since each set of ZY-3 stereo triplet products includes three panchromatic images with different off-nadir angles, 4 stereo pairs can be composed, as shown in Table 3, including one stereo triplet consisting of NAD, BWD, and FWD, and 3 stereoscopic pairs composed of any two images. The accuracy of these stereoscopic pairs in object space is evaluated and compared, and each monoscopic image accuracy in image space is also analyzed and contrasted.

\begin{tabular}{|c|c|c|}
\hline Stereo & Images included & Base/Height \\
\hline Stereo_NBF & NAD, BWD and FWD & 0.88 \\
\hline Stereo_BF & BWD and FWD & 0.88 \\
\hline Stereo_NB & NAD and BWD & 0.44 \\
\hline Stereo_NF & NAD and FWD & 0.44 \\
\hline
\end{tabular}

Table 3. The ZY-3 stereo triplet and stereo pairs

Compared with the absolute geolocation accuracy methodology, the internal geometric accuracy evaluation takes all tiepoints as GCP instead of ICP, and the general workflow is as follows:

1) Bundle adjustment with the GCP to optimize geometry of stereoscopic triplets;

2) Calculate the ground coordinates of the GCP of the stereo triplets or stereo pairs;

3) Calculate the image coordinates of the GCP of the NAD, BWD and FWD triple images;

4) For each GCP, subtract ground reference coordinates from stereo-derived ground coordinates, results in a list of " $\triangle$ Easting", " $\triangle$ Northing" and " $\triangle$ Height" values;

5) For each GCP, subtract image reference coordinates from stereo-derived image coordinates, results in a list of " $\triangle$ Line", and " $\triangle$ Sample" values;

6) For each stereo pair, compute the mean, standard deviations and RMS error of " $\triangle$ Easting", " $\triangle$ Northing" and “ $\triangle$ Height" values;

7) For each image, compute the mean, standard deviations and RMS error of " $\triangle$ Line", and " $\triangle$ Sample" values;

8) Estimate the RMS error statistics for populations of images or stereo pairs, giving the time series analysis of the geometric accuracy of ZY-3 for 8 years.

\subsection{Mono accuracy}

In order to analyze the planimetric accuracy of monoscopic image, the bundle block adjustment with all GCPs is calculated, the internal geometric accuracy of the NAD, BWD and FWD images is evaluated, the magnitude of the error is compared and the change in accuracy is also discussed. 
According to the time series from 2012 to 2019, Figures 8, 9, and 10 depict the horizontal accuracy with GCP of the NAD, BWD, and FWD images, respectively. For the three cases of sample accuracy, line accuracy, and horizontal accuracy, respectively, Figure 11, Figure 12 and Figure 13 compare the differences between NAD, BWD and FWD images.

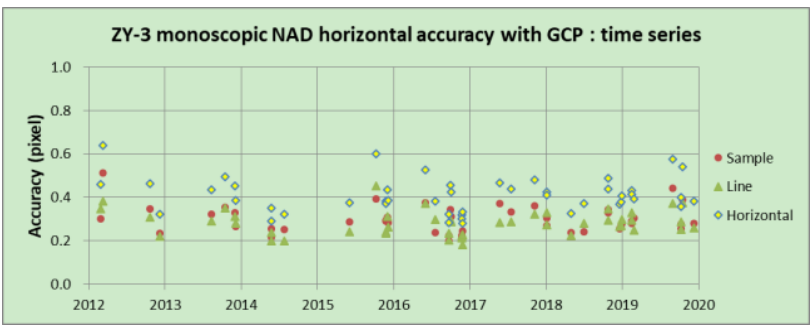

Figure 8. ZY-3 monoscopic NAD horizontal accuracy

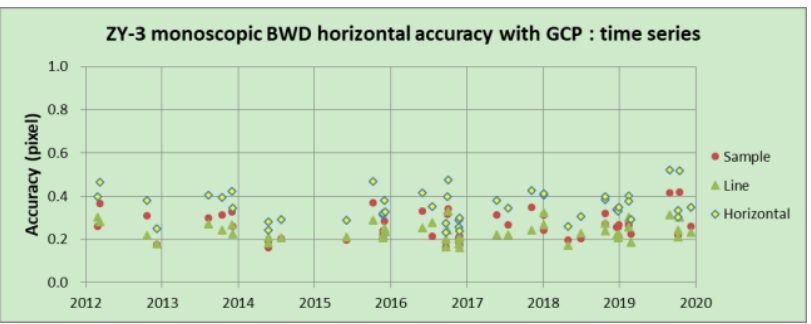

Figure 9. ZY-3 monoscopic BWD horizontal accuracy

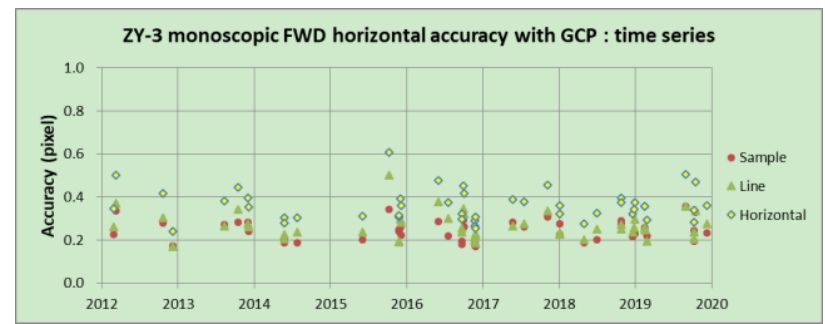

Figure 10. ZY-3 monoscopic FWD horizontal accuracy

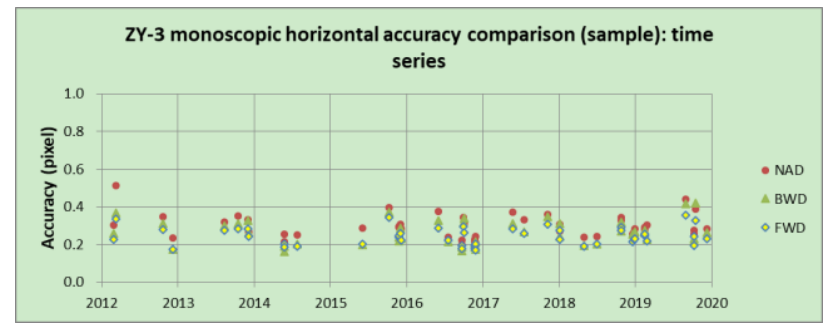

Figure 11. ZY-3 monoscopic horizontal accuracy comparison (sample). Num=47 images; Average errors (sample) of NAD, BWD and FWD are 0.297, 0.262 and 0.243 pixels.

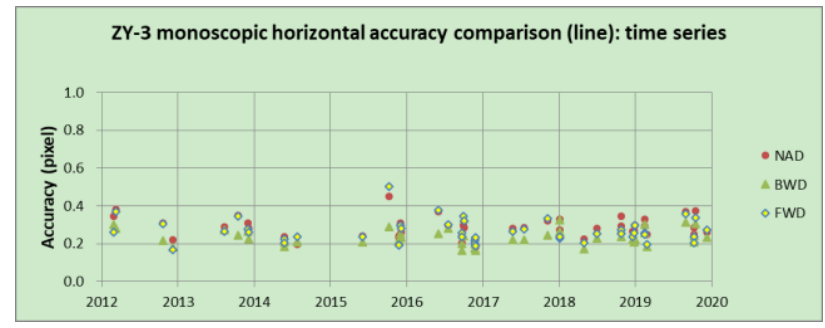

Figure 12. ZY-3 monoscopic horizontal accuracy comparison (line). Num=47 images; Average errors (line) of NAD, BWD and FWD are $0.283,0.236$ and 0.267 pixels.

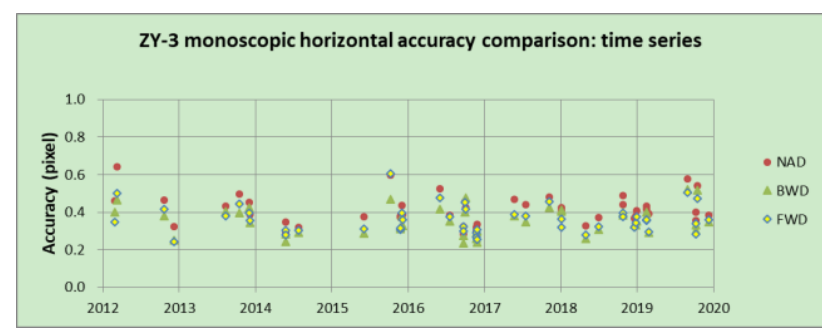

Figure 13. ZY-3 monoscopic horizontal accuracy comparison. Num $=47$ images; Average errors of NAD, BWD and FWD are $0.411,0.354$ and 0.362 pixels, which is equivalent to 0.863 , $1.239,1.2671$ meters.

It can be found from Figures 8 to 13 that overall the internal accuracy of NAD, BWD and FWD images is better than 1.0 pixel, and the accuracy is stable for a long time, which meets the specification requirements. Considering that the GSD of NAD and BWD / FWD are $2.1 \mathrm{~m}$ and $3.5 \mathrm{~m}$, after the accuracy in image space is converted to the ground, they are about $0.863 \mathrm{~m}$, $1.239 \mathrm{~m}$, and $1.267 \mathrm{~m}$, respectively. The accuracy of NAD is significantly better than that of BWD / FWD. There is almost no difference in accuracy between BWD and FWD. In short, these accuracy data are also in line with the expected level.

\subsection{Stereo accuracy}

The stereo accuracy is a very important feature for mapping satellites. The horizontal and vertical accuracy of the four stereo combinations such as Stereo_NBF, Stereo_BF, Stereo_NB and Stereo_NF were evaluated in detail. Especially with regard to planimetric accuracy and elevation accuracy, the differences of each stereos are compared, and their respective advantages are also discussed. According to the time series of up to 8 years, the accuracy changes have also been analyzed.

Fig. 14, Fig. 15, Fig. 16 and Fig. 17 respectively give the geolocation accuracy with GCP of the four stereos such as the Stereo_NBF, Stereo_BF, Stereo_NB and Stereo_NF in accordance with the time series from 2012 to 2019. For each accuracy such as north, east, plenimetric, and elevation, the differences between Stereo_NBF, Stereo_BF, Stereo_NB, and Stereo_NF are shown in Figure 18, Figure 19, Figure 20, and Figure 21.

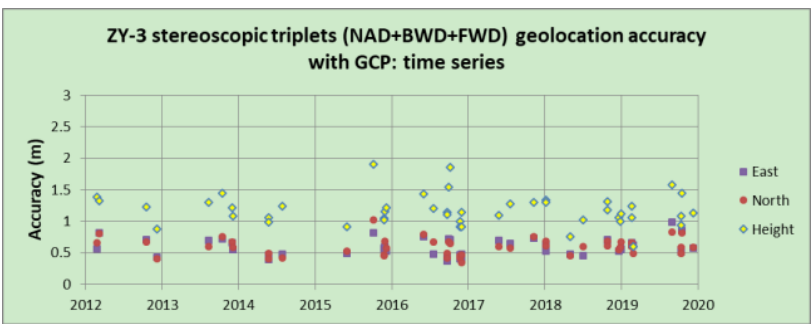

Figure 14. ZY-3 stereoscopic triplets (NAD+BWD+FWD) geolocation accuracy with GCP

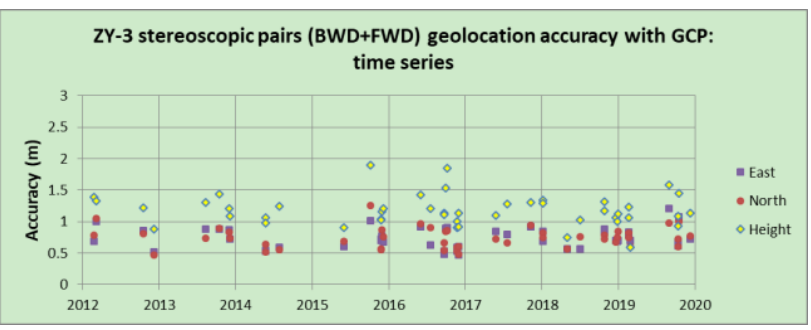

Figure 15. ZY-3 stereoscopic pairs (BWD+FWD) geolocation accuracy with GCP 


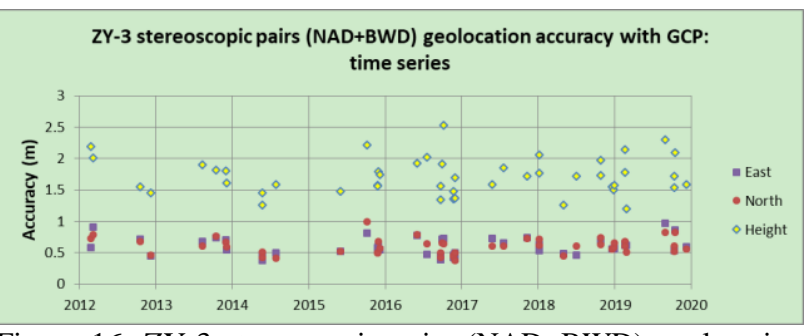

Figure 16. ZY-3 stereoscopic pairs (NAD+BWD) geolocation accuracy with GCP

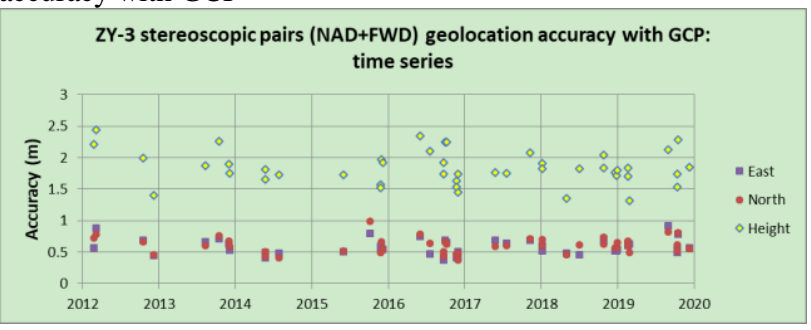

Figure 17. ZY-3 stereoscopic pairs (NAD+FWD) geolocation accuracy with GCP

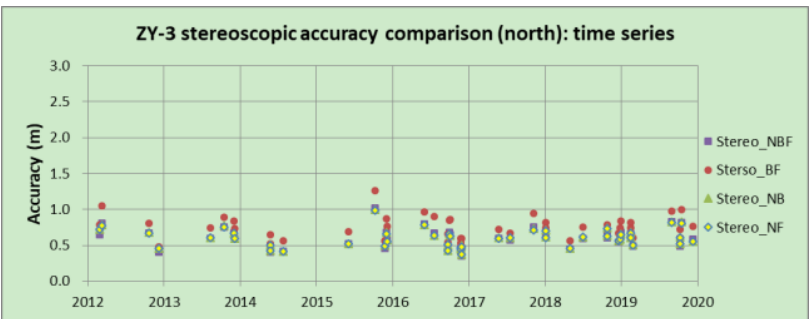

Figure 18. ZY-3 stereoscopic accuracy comparison (north)

Num $=47$ stereo triplets; Average $($ Stereo_NBF $)=0.595 \mathrm{~m}$; Average $($ Stereo_BF $)=0.744 \mathrm{~m} ; \quad$ Average $($ Stereo_NB $)=0.607 \mathrm{~m}$; Average $($ Stereo_NF $)=0.601 \mathrm{~m}$. It can be clearly seen from the figure that the accuracy of Stereo_BF is slightly worse.

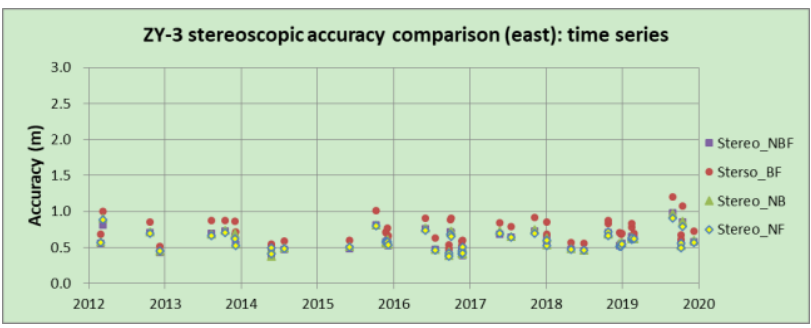

Figure 19. ZY-3 stereoscopic accuracy comparison (east)

Num $=47$ stereo triplets; Average $($ Stereo_NBF $)=0.591 \mathrm{~m}$; Average $($ Stereo_BF $)=0.739 \mathrm{~m} ; \quad$ Average $($ Stereo_NB $)=0.604 \mathrm{~m}$; Average $($ Stereo_NF $)=0.584 \mathrm{~m}$. As shown in the figure, Stereo_BF has poor accuracy.

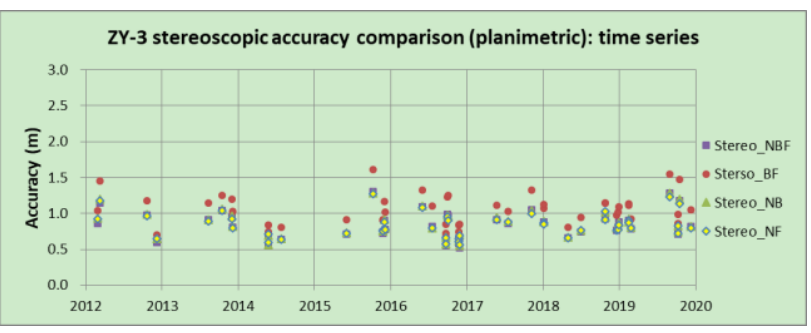

Figure 20. ZY-3 stereoscopic accuracy comparison (planimetric) Num $=47$ stereo triplets; Average $($ Stereo_NBF $)=0.841 \mathrm{~m}$; Average $($ Stereo_BF $)=1.051 \mathrm{~m} ; \quad$ Average $($ Stereo_NB $)=0.857 \mathrm{~m}$; Average $($ Stereo_NF $)=0.840 \mathrm{~m}$. As shown in the figure, the accuracy of Stereo_BF is slightly worse, indicating that adding a higher-precision image to the stereo pair helps improve the planimetric accuracy.

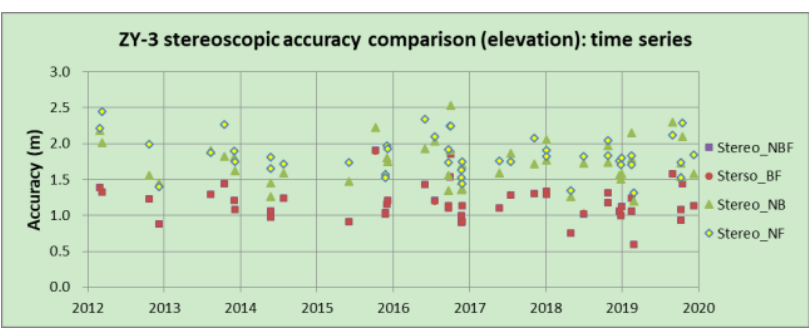

Figure 21. ZY-3 stereoscopic accuracy comparison (elevation) Num $=47$ stereo triplets; Average(Stereo_NBF) $=1.179 \mathrm{~m}$; Average $($ Stereo_BF $)=1.178 \mathrm{~m} ; \quad$ Average $($ Stereo_NB $)=1.723 \mathrm{~m}$; Average(Stereo_NF) $=1.868 \mathrm{~m}$. The elevation accuracy of Stereo_NBF and Stereo_BF is almost the same, there is a phenomenon of overlap. It is obvious from the figure that the elevation accuracy of Stereo_NB and Stereo_NF is lower than that of Stereo_NBF and Stereo_BF, indicating that the elevation accuracy is significantly affected by the base-height ratio.

Summarizing Figures 14 to 21 , it can be shown that the planimetric accuracy and elevation accuracy of Stereo_NBF, Stereo_BF, Stereo_NB, and Stereo_NF are better than 2.0 meters, and continue to be stable for 8 years, which meets the design specifications. In general, for ZY-3 mapping satellite, in the case of flat terrain in this study, the elevation accuracy obtained by Stereo_NBF and Stereo_BF is almost the same, but adding NAD images can slightly improve the planimetric accuracy. For Stereo_NB and Stereo_NF, because the baseheight ratio is much lower than that of Stereo_NBF or Stereo_BF, the elevation accuracy also degrades significantly.

\section{CONCLUSION}

For 8 years, ZY-3 has maintained good direct stereoscopic geolocation performance, the internal geometric accuracy of NAD, BWD and FWD images, the planimetric accuracy and elevation accuracy with GCP, all of which meet design specifications.

Without ground control point, the average planimetric accuracy is approximately $16.2 \mathrm{~m}$ and the average elevation accuracy of about $7.5 \mathrm{~m}$. The internal accuracy of NAD, BWD and FWD images is better than 1.0 pixel, the planimetric accuracy and elevation accuracy of stereo triplets and and stereo pairs are better than 2.0 meters with control points.

The elevation accuracy of Stereo_NBF and Stereo_BF is almost the same in the case of flat terrain in this study. However, the elevation accuracy extracted by Stereo_NB and Stereo_NF will be significantly reduced, because the base-to-height ratio is much lower than that of Stereo_NBF and Stereo_BF.

Together with the good geometric performance, ZY-3 is very suitable for the operational application services of large-scale stereo mapping.

\section{REFERENCES}

Cao Haiyi, Liu Xigang, Li Shaohui and Zhang Xinwei, 2012. ZY-3 Satellite Remote Sensing Technology, Spacecraft Recovery \& Remote Sensing, 33, 7-16.

Foroosh, Hassan, Zerubia, J. B., 2002. Extension of Phase Correlation to Sub-pixel Registration, IEEE Transactions on Image Processing, 11(3), 188-200. 
Grodecki, J., 2001. IKONOS Stereo Feature Extraction - RPC Approach, ASPRS annual conference, St. Louis.

Jacobsen, K., 2007. Orientation of high resolution optical space images, ASPRS annual conference, Tampa.

Kuglin, C. D and Hines D. C., 1975. The Phase Correlation Image Alignment Method, in Proc. Int. Conf. Cybernetics Society, 163-165.

Zhao Liping, Fu Xingke, Dou Xianhui, Liu hui and Fang Zhi, 2018. Comparison and analysis of accuracy of elevation extraction based on the ZY-3 01 and 02 satellites stereoscopic images, Int. Arch. Photogramm. Remote Sens. Spatial Inf. Sci., XLII-3, 2379-2384, doi.org/10.5194/isprs-archives-XLII-32379-2018.

ZY-3A, https://directory.eoportal.org/web/eoportal/satellitemissions/v-w-x-y-z/zy-3a (1 May 2020). 